\title{
The Annals: Welcoming the future of Medicine
}

\author{
Vernon MS $\underline{\mathrm{Oh}},{ }^{1} M D(\mathrm{Camb}), F R C P\left(\right.$ Lond) , Raymond CS $\underline{\operatorname{Seet}}{ }^{1,2}{ }_{M B B S, F R C P(E d i n)}$
}

What started as a cluster of cases of pneumonia in Wuhan, the capital city of China's Hubei Province, in December 2019, has since evolved into a pandemic with devastating consequences to health and livelihoods. ${ }^{1}$ Hardly a day passes without the term COVID-19, the disease caused by the severe acute respiratory syndrome coronavirus 2 (SARS-CoV-2), featuring in mainstream and social media, webinars and conversations.

Once complex viral taxonomy terms are now used freely by policymakers, business analysts, and even school children, as the world braces itself against multiple waves of infections caused by different virus variants, which are named according to the Greek alphabetical notation of Alpha, Beta, Gamma, Delta and, now, Omicron. The pandemic has also compelled the world to put aside previous political differences to deal with a common foe, spawning collaborations between the World Health Organization, governments, and industries to fast-track the development of, and accessibility to, anti-COVID-19 vaccines.

As of 21 December 2021, more than 44\% of the global population has been fully vaccinated (i.e. 2 interval doses) against COVID-19. ${ }^{2}$ The paranoia expressed in stocking of toilet paper has been replaced by a more assured pace of household purchases, while the images of people wearing Hazmat suits in public have been superseded by behavioural changes prioritising physical distancing, frequent hand washing, and face masks. ${ }^{3}$ From being initially shunned, the healthcare workers caring for patients with and without COVID-19 are now shown well-earned gratitude from a grateful public. In Singapore, people encouraged one another by widespread displays of appreciation via coordinated cheers from residents across the country and via the mass media, even when the initial odds of overcoming the dormitory outbreaks and (more recently) the community outbreaks appeared bleak.

Movement restrictions imposed during the pandemic created the ideal setting for healthcare providers to leverage on teleconsultation to reach out to many elderly and vulnerable patients who are homebound, and overseas patients who are no longer able to travel outside their countries. ${ }^{4}$ The need to reopen borders for business travel and families to reconnect after such a long hiatus is greatly facilitated by the deployment of antigen rapid tests (ART) and polymerase chain reaction (PCR) assays at immigration checkpoints. This strategy seeks to avoid bringing in newer cases, as we strive to prevent infection spillage into the community, and to prevent overwhelming our healthcare system.

The Annals has received an unprecedented number of submissions during this pandemic. ${ }^{5}$ We have published several important papers to highlight the impact of the pandemic on the mental well-being of healthcare workers, ${ }^{3,6}$ the clinical management of COVID-19 patients with severe pneumonia, ${ }^{7}$ patterns of emergency department attendances during national lockdowns, ${ }^{8}$ the disease manifestations of pregnant women and children, ${ }^{9}$ short- and long-term sequalae of SARSCoV-2 infection, ${ }^{10,11}$ and finally the rollout experience and adverse effects of COVID-19 vaccination. ${ }^{12}$ At least 3 reviews published in this journal illuminated Singapore's experience in managing the impact of COVID-1913,14 and a policy shift towards handling COVID-19 as an endemic disorder. ${ }^{15}$

Despite the flood of information relating to COVID-19, the Annals has kept up with the other common challenges that Singapore faces in providing quality healthcare to a rapidly ageing population, while ensuring affordable medical costs. ${ }^{16}$ The Annals has published outcomes from the Growing Up in Singapore Towards healthy Outcomes (GUSTO), ${ }^{17}$ the Singapore Mental Health Study, ${ }^{18-20}$ and several other cohort studies providing vital epidemiological insights into disease dynamics in Singapore. Other notable articles covered the cost of managing chronic diseases, ${ }^{16}$ drug utilisation and long-term outcomes of stroke patients, ${ }^{21,22}$ prehospital care among patients with cardiac arrests, ${ }^{23,24}$ risk factors for obstetric emergencies such

\footnotetext{
${ }^{1}$ Department of Medicine, Yong Loo Lin School of Medicine, National University of Singapore, Singapore

${ }^{2}$ Editor-in-Chief, Annals of the Academy of Medicine, Singapore

Correspondence: Assoc Prof Raymond CS Seet, Department of Medicine, Yong Loo Lin School of Medicine, National University of Singapore, NUHS Tower Block, 1E Kent Ridge Road, Singapore 119228.

Email: raymond_seet@nus.edu.sg
} 
as uterine rupture, ${ }^{25}$ Southeast Asia's annual affair with trans-boundary haze, ${ }^{26,27}$ and the adoption of newer laparoscopic and robot-assisted surgical techniques. ${ }^{28}$ To contextualise these reports, we encourage authors to provide bullet-point summaries to explain their key findings and their clinical implications. Content experts are also invited to write leading articles to discuss the pertinence of these topics to Singapore and to the global community.

To commemorate the Annals' 50th anniversary in 2022, we have thoroughly revamped our website (www.annals.edu.sg) to enable quick searches for topics and faster dissemination of information to our growing base of international readers. We have incorporated newer web features to personalise content according to search keywords, and enhance the reader's experience in website navigation. These gains flowed from the migration of our manuscript management to an online system in 2019, which enables the tracking of articles through the different stages of review, decision-making, and copy-editing, and to identify suitable reviewers who can provide an objective evaluation of the merits of the articles. The journal impact factor for the Annals in the 2021 Clarivate Analytics Impact Factor (Web of Science Group) presently stands at 2.473 , ranking it among the leading medical journals in Asia, within the General and Internal Medicine category. Articles published in the Annals are widely cited in academic circles and mainstream media, allowing us ultimately to deliver our primary mission of informing policymakers and healthcare providers of disease and management trends, and to direct funding and philanthropic agencies towards under-investigated areas of emerging importance to Singapore.

The Annals counts on your active contributions to present Singapore's healthcare landscape as we continue to work hard to arrange for high-quality peer reviews and build our reputation as a credible source of information. ${ }^{4}$ As we learn to live with COVID-19, we are required to shift gears to address our healthcare priorities and focus on the basics of disease prevention. We also need to incorporate evidence-based approaches in handling chronic diseases to thwart the onset of complications that often entail costly interventions.

The editorial board and staff at the Annals wish to thank you for your support over the past 50 years, and we look forward to your continued support to take us to greater heights. Sit tight and belt up, as we welcome the future of Medicine!

\section{REFERENCES}

1. Hsu LY, Chia PY, Lim JF. The Novel Coronavirus (SARS-CoV-2) Epidemic. Ann Acad Med Singap 2020;49:105-7.

2. World Health Organization. WHO Coronavirus (COVID-19) Dashboard. Available at: https://covid19.who.int/table. Accessed on 22 December 2021.

3. Ho CS, Chee CY, Ho RC. Mental Health Strategies to Combat the Psychological Impact of Coronavirus Disease 2019 (COVID-19) Beyond Paranoia and Panic. Ann Acad Med Singap 2020;49:155-60.

4. Wang LY, Low TT, Yeo TJ. Telehealth in COVID-19 and Cardiovascular Disease-Ensuring Equitable Care. Ann Acad Med Singap 2020;49:902-4.

5. Oh VM, Seet RC. The Annals and the Medical Narrative of Singapore. Ann Acad Med Singap 2020;49:103-4.

6. Tan MY, Rajgor DD, Heng CK, et al. Stress and resilience of paediatric healthcare workers during COVID-19. Ann Acad Med Singap 2021;50:339-42.

7. Puah SH, Cove ME, Phua J, et al. Association between lung compliance phenotypes and mortality in COVID-19 patients with acute respiratory distress syndrome. Ann Acad Med Singap 2021;50:686-94

8. Cheng L, Ng WM, Lin Z, et al. Factors reducing inappropriate attendances to emergency departments before and during the COVID-19 pandemic: A multicentre study. Ann Acad Med Singap 2021;50:818-26

9. Li J, Thoon KC, Chong CY, et al. Comparative Analysis of Symptomatic and Asymptomatic SARS-CoV-2 Infection in Children. Ann Acad Med Singap 2020;49:530-7.

10. Li TYW, Ngiam JN, Chew NWS, et al. Low incidence of cardiac complications from COVID-19 and its treatment among hospitalised patients in Singapore. Ann Acad Med Singap 2021;50:490-3.

11. Mitra S, Ling RR, Yang IX, et al. Severe COVID-19 and coagulopathy: A systematic review and meta-analysis. Ann Acad Med Singap 2021;50:325-35.

12. Tan JTC, Tan C, Teoh J, et al. Adverse reactions and safety profile of the mRNA COVID-19 vaccines among Asian military personnel. Ann Acad Med Singap 2021;50:827-37.

13. Tan THY, Toh M, Vasoo S, et al. Coronavirus Disease 2019 (COVID-19): The Singapore Experience. A Review of the First Eight Months. Ann Acad Med Singap 2020;49:764-78.

14. Chen JI, Yap JC, Hsu LY, Teo YY. COVID-19 and Singapore: From Early Response to Circuit Breaker. Ann Acad Med Singap 2020;49:561-72.

15. Wei WE, Tan WK, Cook AR, et al. Living with COVID-19: The road ahead. Ann Acad Med Singap 2021;50:619-28.

16. Tan SY, Lew KJ, Xie Y, et al. Healthcare cost of patients with multiple chronic diseases in Singapore public primary care setting. Ann Acad Med Singap 2021;50:809-17.

17. Tan KML, Chu AHY, Loy SL, et al. Association of Cord Blood Thyroid-Stimulating Hormone Levels with Maternal, Delivery and Infant Factors. Ann Acad Med Singap 2020;49:937-47.

18. Subramaniam M, Lau JH, Abdin E, et al. Impact of unemployment on mental disorders, physical health and quality of life: Findings from the Singapore Mental Health Study. Ann Acad Med Singap 2021;50:390-401.

19. Lee YY, Lau JH, Seet V, et al. Dietary intake of persons with depressive and psychotic disorders in Singapore. Ann Acad Med Singap 2021;50:379-89. 
20. Subramaniam M, Abdin E, Vaingankar J, et al. Obsessive-Compulsive Disorder in Singapore: Prevalence, Comorbidity, Quality of Life and Social Support. Ann Acad Med Singap 2020;49:15-25.

21. The EZ, Ng MY, Ng GJ, et al. Long-term outcomes of ischaemic stroke patients with diabetes in a multi-ethnic cohort in Singapore. Ann Acad Med Singap 2021;50:16-25.

22. Tang TY, Patel A, Soon SXY, et al. Improving medical adherence and antithrombotic management for patients with chronic limb threatening ischaemia in Singapore. Ann Acad Med Singap 2021; 50:795-7.

23. Ho AF, Lee KY, Lin X, et al. Nation-Wide Observational Study of Cardiac Arrests Occurring in Nursing Homes and Nursing Facilities in Singapore. Ann Acad Med Singap 2020;49:285-93.

24. Tay WJ, Li H, Ho AF, et al. Mortality and Neurological Outcomes in Out-of-Hospital Cardiac Arrest Patients With and Without
Targeted Temperature Management in a Multiethnic Asian Population. Ann Acad Med Singap 2020;49:127-36.

25. Tan SQ, Chen LH, Muhd Abdul Qadir DB, et al. Risk factors and outcomes of uterine rupture in Singapore: Emerging trends. Ann Acad Med Singap 2021;50:5-15.

26. Chan SL, Ho AF, Ding $\mathrm{H}$, et al. Impact of Air Pollution and Trans-Boundary Haze on Nation-Wide Emergency Department Visits and Hospital Admissions in Singapore. Ann Acad Med Singap 2020;49:78-87.

27. Ng KYY, Yeung W, Sou KL, et al. Factors influencing protective behaviours during haze episodes in Singapore: A populationbased study. Ann Acad Med Singap 2021;50:514-26.

28. Goh BK, Wang Z, Koh YX, Lim KI. Evolution and trends in the adoption of laparoscopic liver resection in Singapore: Analysis of 300 cases. Ann Acad Med Singap 2021;50:742-50. 